\title{
Formação de pesquisadores em Etnomatemática: a experiência do GETUFF
}

\author{
Maria Cecilia Fantinato ${ }^{1}$ \\ José Ricardo e Souza Mafra² \\ Claudia de Jesus Meira ${ }^{3}$
}

\section{RESUMO}

Este texto relata a experiência do Grupo de Etnomatemática da UFF (GETUFF), com foco na formação em pesquisa. Trata-se de um artigo escrito por três pessoas, que relatam sua experiência de coformação no contexto do Grupo, a partir de lugares diferenciados: da coordenadora e fundadora do grupo, da doutoranda que participa ativamente do GETUFF há cerca de seis anos e do pesquisador visitante que realizou pós-doutorado em 2015 sob supervisão da coordenadora. O artigo traz as transformações do Grupo ao longo do tempo e as experiências de formação vividas por cada autor (a) neste contexto. Ao final traz algumas reflexões sobre a importância dos grupos de pesquisa na formação de professores e pesquisadores em Etnomatemática.

PALAVRAS-CHAVE: Etnomatemática. Formação de pesquisadores. Grupos de pesquisa.

Training of researchers in Ethnomathematics: the experience of GETUFF

\begin{abstract}
This text reports the experience of the Ethnomathematics Group of UFF (GETUFF), focusing on research training. It is an article written by three people, who report their experience of co-formation in the context of the Group, from different places: from the coordinator and founder of the group, from the doctorate who has actively participated in GETUFF for about six years and the researcher who underwent postdoctoral studies
\end{abstract}

\footnotetext{
${ }^{1}$ Doutora em Educação. Universidade Federal Fluminense, Niterói/RJ, Brasil. E-mail: mcfantinato@gmail.com ${ }^{2}$ Doutor em Educação. Universidade Federal do Oeste do Pará, Santarém/PA, Brasil. E-mail: jose.mafra@ufopa.edu.br

${ }_{3}^{3}$ Mestre em Educação. Universidade Federal Fluminense, Niterói/RJ, Brasil. E-mail: claumeira1976@gmail. com
} 
under the supervision of the coordinator in 2015. The article brings the transformations of the Group over time and the experiences of formation experienced by each author in this context. At the end, it brings some reflections on the importance of research groups in the training of teachers and researchers in Ethnomathematics.

KEYWORDS: Ethnomathematics. Training of researchers. Research groups.

\section{Introdução}

Desde o surgimento da Etnomatemática como tendência da Educação Matemática, na década de oitenta, várias têm sido as estratégias de disseminação e consolidação desta linha de estudos e pesquisas, como afirma Passos (2017):

Os periódicos, a formação acadêmica, os eventos científicos, os grupos de pesquisa e as redes de relações estabelecidas pelas orientações foram estratégias tomadas em nosso texto como referência para que, em um primeiro momento, nos fornecesse um panorama geral da etnomatemática, mostrando ser esta uma área de pesquisa reconhecida e legitimada do campo acadêmico (PASSOS, 2017, p. 144).

Os grupos de pesquisa, em particular, têm exercido um importante papel neste movimento de fortalecimento da área. Uma das funções importantes desses grupos é a formação de pesquisadores.

Este texto vem, portanto, relatar a experiência do Grupo de Etnomatemática da UFF (GETUFF), com foco na formação em pesquisa. Trata-se de um artigo escrito por três pessoas, que relatam sua experiência de coformação no contexto do Grupo, a partir de lugares diferenciados: da coordenadora e fundadora do grupo, da doutoranda que participa ativamente do GETUFF há cerca de seis anos e do pesquisador visitante que realizou pós-doutorado em 2015 sob supervisão da coordenadora. Optamos 
então por seguir esta sequência cronológica na escrita do artigo, que retrata bem o crescimento do GETUFF ao longo desses quase catorze anos de existência. Fechamos trazendo algumas considerações sobre a importância dos grupos de pesquisa na formação de professores e pesquisadores em Etnomatemática.

\section{A construção coletiva do GETUFF}

O Grupo de Etnomatemática da UFF (GETUFF) foi criado em setembro de 2004, na Faculdade de Educação da UFF, a partir das discussões surgidas durante um minicurso ministrado por Maria Cecilia Fantinato, que era parte das atividades de um curso de extensão em parceria da UFF com o Programa de Educação de Jovens e Adultos (PEJA), da Secretaria Municipal do Rio de Janeiro. Seus primeiros integrantes eram, portanto, professores da rede pública de ensino e pesquisadores, interessados nas contribuições da Etnomatemática para a prática docente, sobretudo da EJA. O perfil inicial foi aos poucos se modificando, passando a incorporar também, além dos professores das redes de Educação Básica, estudantes de Graduação e Pós-Graduação e outros professores de universidades do Estado do Rio de Janeiro. "Desde então, tem primado por ser um espaço pioneiro de estudos e de pesquisa na área da Etnomatemática no Estado do Rio de Janeiro”. (THEES et al, 2013, p. 7926).

Desde sua formação, havia entre os integrantes, de níveis de formação ou inserção profissional tão diversos, o interesse comum em aprofundar os estudos na área da Etnomatemática, assim como a possibilidade de participar de um espaço de discussão sobre questões teórico-práticas ligadas à educação matemática. Inicialmente os estudos do Grupo eram dirigidos a reflexões teóricas que auxiliassem na delimitação da área, assim como quanto na busca por elementos que possibilitassem desenvolver um trabalho em Etnomatemática no cotidiano das salas, especialmente, da Educação de Jovens e Adultos (EJA). Algumas das temáticas abordadas neste período 
eram as relações da Etnomatemática com: a EJA, a Prática Pedagógica, a Formação de Professores, a Educação Indígena e as Etnociências.

A dinâmica de encontros do Grupo também foi se transformando ao longo do tempo. Assim que percebemos que os encontros quinzenais dos primeiros anos eram insuficientes para nossas demandas internas, passamos a nos reunir semanalmente, sempre em alguma sala da Faculdade de Educação de UFF, em Niterói. A inserção da coordenadora do grupo no Programa de Pós-Graduação em Educação da UFF (PPGE-UFF), a partir de 2008, também trouxe um aumento efetivo de número de alunos mestrandos, doutorandos e professores mestres ou doutores no grupo. Mas a característica de grupo colaborativo, um espaço de troca de saberes e de experiências, onde "todos trabalham conjuntamente (co-laboram) e se apoiam mutuamente, visando atingir objetivos comuns negociados pelo coletivo do grupo" (FIORENTINI, 2004, p. 50), se manteve ao longo dos anos.

Nos primeiros anos do GETUFF, as atividades desenvolvidas consistiam:

[...] principalmente ao estudo de textos, debates e organização de palestras, com convidados da própria UFF ou de outras instituições, de áreas diretamente relacionadas à Etnomatemática ou não, que pudessem contribuir para as reflexões do grupo. $\mathrm{O}$ grupo também participava de atividades de formação continuada de professores em projetos da FEUFF (THEES et al, 2013, p. 7927).

A principal realização do Grupo, naquele período, foi a organização do Terceiro Congresso Brasileiro de Etnomatemática (CBEm3) em março de 2008, na Faculdade de Educação da UFF. O livro Etnomatemática: novos desafios teóricos e pedagógicos (FANTINATO, 2009) foi um dos resultados deste evento.

Nos últimos anos, as discussões do GETUFF têm priorizado o aprofundamento de questões teórico-metodológicas oriundas das pesquisas 
desenvolvidas por seus integrantes, sendo uma das atividades recorrentes a discussão dos projetos dos mestrandos ou candidatos ao mestrado ou doutorado do Programa de Pós-Graduação em Educação da UFF. Também têm sido propostas leituras de textos da produção acadêmica da Etnomatemática ou de áreas afins. A organização de Seminários do GETUFF com pesquisadores convidados, abertos ao público externo e divulgados pela página do PPGE-UFF assim como pelas redes sociais, têm ampliado a riqueza do debate e têm se constituído em oportunidades de socialização de nossos estudos e discussões. Todas essas atividades têm gerado frutos, aumentando a produção do Grupo, principalmente na forma de trabalhos apresentados em eventos científicos ou em periódicos qualificados, de forma individual ou, sobretudo, em coautoria de dois ou mais integrantes.

Em setembro de 2014 aconteceu na Faculdade de Educação da UFF o Encontro de Etnomatemática do Rio de Janeiro (ETNOMAT-RJ), como um evento satélite do VI Encontro Estadual de Educação Matemática do Rio de Janeiro (VI EEMAT). A realização deste evento, destinado a toda comunidade brasileira de pesquisadores e professores interessados na Etnomatemática, tinha como objetivo comemorar os dez anos de existência do Grupo de Etnomatemática da UFF, propondo um exercício de reflexão sobre a trajetória percorrida pelos grupos de pesquisa na área, sinalizando para novas perspectivas. Os trabalhos enviados deveriam se inserir em um dos eixos temáticos sugeridos pela organização para envio dos trabalhos, a saber: Diálogos da Etnomatemática com a Educação e aspectos teóricos e filosóficos da Etnomatemática.

O trabalho de organização coletiva do ETNOMAT-RJ implicou em uma série de atividades formativas. Com efeito, as decisões sobre os eixos temáticos, a leitura e a classificação dos resumos dos trabalhos para a organização das salas de apresentação durante o evento, além de todas as tarefas operacionais desempenhadas pelo GETUFF, possibilitaram a seus integrantes oportunidade única de amadurecimento acadêmico e de 
discussão sobre a pesquisa em Etnomatemática. Tal experiência nos instigou a dar continuidade ao esta procura de compreensão ampla da produção da área, pelo estudo dos Anais do ETNOMAT-RJ. Foi desenvolvida então uma pesquisa bibliográfica do tipo estado da arte (FERREIRA, 2002), visando mapear e discutir a produção acadêmica em nosso campo do conhecimento, a partir do recorte dos trabalhos publicados nos Anais do evento. Esta investigação, desenvolvida coletivamente em todas as suas etapas entre 2015-2017 e visando a publicação de um livro do Grupo, envolveu diversos saberes e fazeres, elaborados durante todo processo.

Os resultados finais deste trabalho foram publicados no livro Etnomatemática: concepções, dinâmicas e desafios (FANTINATO \& FREITAS, 2018).

\section{A experiência no GETUFF do ponto de vista de uma doutoranda}

Fazer parte do corpo discente de um programa de pós-graduação, talvez seja o desejo de muitos concluintes da graduação, mas se descobrir um pesquisador de uma área específica, requer do mestrando/doutorando um processo intenso de amadurecimento e autonomia.

Neste processo, que muitas vezes é marcado por produções, prazos, isolamentos e interações, fazer parte de um grupo de pesquisa é um passo relevante na formação do pesquisador iniciante onde este tem:

a oportunidade de inserção num projeto coletivo, em que seja possível (com)partilhar de um referencial teórico comum e ao mesmo tempo, desenvolver, apoiado pelo grupo um ângulo específico de uma problemática mais ampla, o trabalho final poderá vir a atingir um nível de qualidade melhor do que se feito isoladamente (ANDRÉ, 2007, p. 50).

Sob esta ótica, a participação em um grupo de pesquisa pode lhe proporcionar ao futuro pesquisador o devido amadurecimento no que diz 
respeito a questões concernentes a sua pesquisa como um aprofundamento no aprendizado teórico/empírico e nos procedimentos metodológicos, oferecendo assim uma contribuição essencial nas produções científicas de estudantes dos programas de pós-graduação.

A seguir pretendemos fazer um breve relato do encontro e o que foram as intervenções do GETUFF em nossa formação enquanto mestranda do programa de Pós-graduação em Educação da UFF.

Encontrar pares dispostos a dialogar sobre o que D’Ambrosio (2005, p. 102) classificou como "estudo da evolução cultural da humanidade no seu sentido amplo, a partir da dinâmica cultural que se nota nas manifestações matemáticas" nos pareceu uma árdua tarefa. Nesta busca nos enveredamos pelos caminhos da história das Ciências, técnicas e epistemologia (UFRJ 2007), mas logo fomos aconselhados a buscar pares na Etnomatemática. Finalmente em curso de extensão para professores de matemática na UFF, veio a orientação e a indicação para conhecer a professora Fantinato e, por conseguinte o GETUFF, um grupo hospitaleiro, crítico, diverso em sua composição e formação, mas convergindo no que diz respeito a "entender o fazer e o saber matemático de culturas marginalizadas" (D’AMBROSIO, 2004, p. 44).

$\mathrm{Na}$ condição de mestranda em 2013, nosso objetivo era analisar os saberes/fazeres de jovens e adultos em contexto de privação de liberdade sob a perspectiva da Etnomatemática (MEIRA, 2015). Nesse caminho nos deparamos com o desafio da ausência de literatura específica que tratasse das questões da Etnomatemática em um contexto inóspito de uma prisão.

Estávamos diante de um problema que acreditamos ser relevante e original para a área e devia ser pesquisado, tínhamos muitas questões de ordem metodológica e na literatura encontramos alguns caminhos, segundo Lüdke e André (1986) para a realização de uma pesquisa:

[...] é preciso promover o confronto entre os dados, as evidências, as informações coletadas sobre determinado assunto e 
conhecimento teórico adquirido a respeito dele. Em geral isso se faz do estudo de um problema que ao mesmo tempo desperta o interesse de um pesquisador e limita sua atividade de pesquisa a uma determinada porção do saber, a qual ele se compromete a construir naquele momento (LÜDKE e ANDRÉ, 1986, p. 2).

Como professora de uma escola inserida em contexto prisional, tínhamos afinidade e proximidade com o contexto onde a pesquisa seria realizada, mas faltava o olhar acadêmico que nos permitisse observar o fenômeno, delimitar um tema/problema e as nuances pertinentes da pesquisa científica, delineadas pelas autoras.

A inserção em um grupo de pesquisa - o GETUFF - constituiu-se em uma atividade de interação, reflexão e inquietação, com o intuito de construir novos conhecimentos que serviriam na resolução não só de nosso problema pontual da pesquisa, mas também de outros colegas que também estavam envolvidos no processo coletivo de aprendizagem, fazendo-nos entender o caráter social do trabalho, o que foi de extrema relevância para nosso desenvolvimento enquanto pesquisadora.

Tínhamos um grande desafio com a pesquisa: entrelaçar e formar uma base teórica sólida para escrevermos sobre Educação de Jovens e Adultos em privação de liberdade e Etnomatemática com a prisão como pano de fundo.

Os estudos metódicos reflexivos no GETUFF de textos como os de Ubiratan D’Ambrósio, Darlinda Moreira, Maria do Carmo Domite, Reinaldo Fleuri, Maria Conceição Fonseca, Barbara Rogoff, Jean Lave e Etienne Wenger entre outros autores, nos ofereceram subsídios para organizar um referencial teórico estruturado para o nosso texto dissertativo.

Em grupo também analisamos as produções recentes de nossos colegas de GETUFF e de outros pesquisadores, o que nos trouxe um norte para escolha metodológica de nossa pesquisa.

Da experiência coletiva de análise de textos, nos encaminhamos para o percurso solitário e necessário do pesquisador, com o fim de redefinir nossas 
escolhas e especificamente em nossa pesquisa de mestrado, assumirmos um posicionamento quanto à abordagem escolhida: qualitativa e de cunho etnográfico. Nosso objetivo era fazer a descrição profunda do contexto, apreendendo seus significados da cultura local, e a partir dos dados adquiridos, reapresentá-los como um novo significado aos indivíduos externos ao contexto (BOGDAN e BIKLEN, 1994).

Tais escolhas nos possibilitaram compreender que:

a abordagem etnográfica nos permite conhecer o aluno privado de liberdade em meio às suas práticas considerando sua heterogeneidade e sua diversidade sociocultural e com as lentes da Etnomatemática vislumbrar nestas práticas saberes impregnados de conhecimentos matemáticos relacionados à comparação, inferição, generalização, medição, classificação, quantificação [...] (MEIRA, 2015, p. 43).

A partir daí seguimos, agora mais seguros, apostando na hipótese inicial da possibilidade de diálogos entre os saberes/fazeres de jovens adultos em privação de liberdade e as aulas de matemáticas, no processo de levantamento de dados para a pesquisa.

Ao longo do processo, nossa formação também foi enriquecida através de visitas de pesquisadores externos ao programa nas reuniões do GETUFF, tais como Carmem Cavaco (UL-PT) ${ }^{4}$ que nos apresentou seu trabalho de formação experiencial em adultos pouco escolarizados; Darlinda Moreira $(\mathrm{UAb}-\mathrm{PT})^{5}$ que nos apresentou seu trabalho de aprendizagem ao longo da vida como uma tendência atual e global; José Ricardo Mafra (UFOPA) ${ }^{6}$ nos apresentou os artefatos das ribeirinhas de Aritapera-PA em seu trabalho pós doutoral; Sonia De Vargas (UCP) 7 nos presenteou com sua experiência

\footnotetext{
${ }^{4}$ Universidade de Lisboa - Portugal.

${ }^{5}$ Universidade Aberta - Portugal.

${ }^{6}$ Universidade Federal do Oeste do Pará.

${ }^{7}$ Universidade Ctólica de Petrópolis.
} 
na Educação de Jovens e Adultos e Sonia Clareto (UFJF)8 esteve conosco para conversar sobre as possibilidades filosóficas em Etnomatemática.

Todos esses pesquisadores nos alertaram para as diversas possibilidades que uma pesquisa em Etnomatemática podem apontar, assim como para as suas dimensões ${ }^{9}$, contribuindo com nosso referencial teórico e nossas escolhas na produção do texto final.

Além disso o GETUFF nos ofereceu a possibilidade de participação em produções textuais para eventos científicos nacionais e internacionais ${ }^{10}$. Nosso primeiro evento representando o GETUFF foi o IIV CIBEM em 2013, onde em companhia de colegas do grupo apresentamos o trabalho Retrospectiva e perspectivas do Grupo de Etnomatemática da UFF GETUFF e também apresentamos nosso trabalho de forma individual, já um ensaio para nosso exame de projeto que se realizaria no retorno desta viajem.

Para Campello (2000) os eventos científicos:

podem desempenhar diversas funções: encontros como forma de aperfeiçoamento de trabalhos científicos, uma vez que os trabalhos apresentados mudam substancialmente após apreciação nos eventos; encontro como reflexo do estado da arte, pois os trabalhos apresentados durante os eventos podem refletir o panorama da área e o perfil dos seus membros e encontros como forma de comunicação informal, pois as conversas informais com seus pares constituem parte importante dos eventos. (CAMPELLO, 2000, p. 62 apud LACERDA et al., 2008, p. 130-144).

Hoje entendemos que é por meio desses eventos que os trabalhos científicos são aperfeiçoados, ao receberem apreciações, sugestões, críticas, havendo também, uma troca de ideias e fatos novos. Sem isso, entendemos

\footnotetext{
${ }^{8}$ Universidade Federal de Juiz de Fora.

${ }^{9}$ As dimensões da Etnomatemática: conceitual, histórica, cognitiva, epistemológica, política e educacional. Maiores detalhes D’Ambrosio, U. Etnomatemática - Elo entre tradições e modernidade. Belo Horizonte: Autentica, 2001.

${ }^{10}$ IIV CIBEM (Uruguai), ICEM5 (Moçambique), ANPED (Florianópolis) entre outros.
} 
que as condições de avaliação da qualidade, a repercussão e a aceitação do conhecimento produzido ficariam difíceis.

Através do GETUFF também fizemos parte da comissão organizadora do ETNOMAT-RJ, um encontro que, além da comemoração dos dez anos de existência de nosso grupo, objetivou congregar professores e pesquisadores em Etnomatemática. Inicialmente com pretensões regionais, chegamos a receber pesquisadores de todas as regiões do Brasil e exterior. Nas reuniões de organização deste evento, agregamos à nossa formação experiência nesta área assim como um estreitamento nas interações com pesquisadores mais experientes da área.

Com a conclusão do mestrado e ingresso no doutorado, passamos a entender a relevância das ações coletivas do grupo de pesquisa em nossa formação discente. A participação efetiva no GETUFF contribui permanentemente com o enriquecimento da área e do pesquisador, complementando a sua formação e o seu conhecimento. Esta propicia um maior convívio no ambiente acadêmico e desperta no estudante um maior envolvimento, participação, troca de ideias e experiências, além de uma visão mais globalizada de conhecimento, os quais são fundamentais para sua profissão.

Também, é possível aos estudantes adquirir através da divulgação de suas pesquisas e seus relatos de experiências avaliações, críticas e sugestões para a melhoria dos mesmos. Além disso, há a possibilidade de pôr em prática o conhecimento adquirido.

Atualmente como doutoranda, o grupo tem nos propiciado a oportunidade de participar em novos empreendimentos coletivos e individuais. Os encaminhamentos e experiência empírica da pesquisa realizada no mestrado tem nos direcionado a reflexões voltadas ao campo teórico da Etnomatemática, tema ainda em construção, mas certa que o GETUFF é um potencializador de mais este estudo. 


\section{Experiências formativas, na perspectiva de um pós-doutorando}

Esta seção visa apresentar um breve relato das experiências adquiridas, no período de um ano de realização do projeto de pós-doutorado intitulado Artesãs de Aritapera/PA: técnicas e processos evidenciados em cuias tapajônicas, submetido ao Programa de Educação/UFF. O texto traça um panorama das etapas de implementação e de desenvolvimento da proposta de pesquisa, desenvolvidas ao longo de 2015, sob a supervisão de Maria Cecilia Fantinato, docente permanente da Universidade Federal Fluminense e vinculada ao Programa de Pós-graduação em Educação/UFF.

As atividades de pós-doutorado foram desenvolvidas na UFF, durante o ano de 2015 e 2016 e envolveram uma série de ações decorrentes do plano de metas estipulado no início do estágio pós-doutoral, a saber:

i) participação em bancas de qualificação. Durante as atividades de pós-doutorado foi possível a participação em bancas de qualificação de mestrado, como examinador externo. O trabalho de Meira (2015) e Cruz (2016), trabalhos estes orientados por Maria Cecilia Fantinato e vinculados ao PPGE/UFF, até então, em desenvolvimento, forneceram uma base de discussão significativa para os nossos processos formativos.

ii) participação em reuniões frequentes no Grupo de Pesquisa em Etnomatemática da UFF - GETUFF. As atividades referentes à participação no GETUFF ocorreram entre os meses de fevereiro e dezembro de 2015 , sempre às $6^{\mathrm{a}}$ feiras, das $9 \mathrm{~h}$ às $12 \mathrm{~h}$. Durante o desenvolvimento dos encontros do grupo de pesquisa tivemos a oportunidade de apresentar e socializar, além de nossa proposta de pesquisa, temáticas de discussões inseridas em textos e artigos selecionados e vinculados a temas relacionados à área de inquérito da Etnomatemática.

iii) disciplinas ministradas na graduação e pós-graduação. Foram desenvolvidas atividades nas disciplinas de graduação: Matemática: Conteúdo e Método I, vinculada ao curso de Pedagogia/UFF e a organização, planejamento e execução, da disciplina de pós-graduação Tópicos Especiais 
em DDSE: Elementos Teóricos e Metodológicos para as Pesquisas em Etnomatemática.

O desenvolvimento das disciplinas contribuiu para a base de reflexões teóricas assumidas durante os estudos realizados e, quando contrastados com as informações advindas da pesquisa de campo, estabeleceram-se contribuições, não apenas para os estudos de pós-doutorado, mas também, para a assunção de uma interlocução possível com base em processos e saberes informais. Esses saberes estavam imersos em experiências educacionais, cujo foco de estudos de mestrado e doutorado, faziam parte do objeto de pesquisa dos participantes matriculados na disciplina.

iv) atividades de discussão e reflexão conjunta entre supervisionado e supervisora sobre referenciais e estudos teóricos envolvendo a temática relativa a pesquisa. Durante o desenvolvimento da investigação ocorreram reuniões sistematicas para o delineamento da pesquisa e planejamento das ações metodológicas e organização de uma sustentação teórica inicial, tendo em vista a pesquisa etnográfica que ocorreu ao longo do ano de 2015. Tais discussões contribuiram significativamente para uma compreensão maior sobre o campo de investigação da pesquisa em desenvolvimento, até então, pois, agregou-se aos estudos realizados, para além dos referenciais em Etnomatemática, estudos organizados de acordo com elementos da pesquisa antropológica e social. Mais especificamente, como as conexões existentes entre os saberes informais e as ideias matemáticas associadas, fornecem indicativos ou indícios da construção de uma ponte que permita a disseminação de propostas educacionais para o trabalho envolvendo características intrínsecas ao saber matemático (CHAMOUX, 1978; GREENFIELLD, 1999; DASEN, 2004; LAVE \& WENGER , 1993).

v) participação em eventos, tais como palestras, conferências e oficinas. A participação em atividades inerentes a pesquisa acadêmica se tornou uma constante durante todo o processo de estudos, no nível de pós-doutorado, de forma a apresentar os resultados da pesquisa, então em curso. 
vi) desenvolvimento da pesquisa vinculada ao projeto inicial. Este estudo, intitulado Artesãs de Aritapera/PA: técnicas e processos evidenciados em cuias tapajônicas, teve como principal objetivo a busca por uma interface entre saberes informais, centrados na produção laboral das artesãs e como eles se interconectam com a perspectiva do Programa Etnomatemática. Foi desenvolvido na busca por uma compreensão sobre processos, técnicas e estratégias de saber fazer, utilizados por um grupo de mulheres artesãs, residentes na cidade Santarém/PA, Região do Aritapera.

Foi pautado em referenciais advindos da Antropologia e da Etnomatemática (D’AMBROSIO, 2001; MOREIRA \& PIRES, 2012), na busca por entender como as culturas mais diversas expõem e disseminam suas razões e explicações resultantes - a partir das interações com o meio de sobrevivência.

vii) produtos gerados, decorrentes das informações obtidas no campo de investigação, tais como: artigos em periódicos, artigos completos em anais de eventos. A proposta de investigação, bem como a participação efetiva nas atividades do GETUFF permitiram a produção de diversos trabalhos. Os mais relevantes, tratam da investigação propriamente dita (MAFRA, 2015; MAFRA \& FANTINATO, 2016 e FANTINATO \& MAFRA, 2017).

As atividades desenvolvidas durante o período do pós-doutorado se mostraram muito profícuas, em diversos pontos. Um destes pontos trata da constituição de grupo de estudos, colaboração e de pesquisa internacional. Este grupo foi constituído durante as atividades do ICME-13 em Hamburgo, no ano de 2016, e conta com a participação de dois docentes do Brasil, uma docente da Bélgica e um docente de França. Assumiu-se a importância e necessidade de um intercâmbio maior, em termos de socialização de pesquisas desenvolvidas em diferentes regiões do planeta, o que levou a formação do grupo de trabalho.

Os resultados iniciais deste grupo de trabalho estão registrados em dois trabalhos produzidos: um, durante o ano de 2017 (VANDENDRIESSCHE et al., 2017), apresentando elementos de discussão 
teórica, a partir de duas pesquisas empíricas, baseadas em investigações etnográficas e outro, recentemente publicado (FRANÇOIS et al., 2018), na qual traz uma contribuição em relação a perspectivas pedagógicas possíveis, em relação aos estudos envolvendo práticas socioculturais, tendo por base ideias matemáticas e antropológicas, localizadas em diferentes contextos de investigação.

É importante destacar que, a produção e discussão associada ao grupo de estudo internacional, só começou a ser efetivamente concretizada, a partir das reuniões realizadas durante a ocorrência do ICME-13, ponto de partida para discussões e a elaboração conjunta de trabalhos que culminaram com as publicações indicadas nos dois parágrafos anteriores.

Além das atividades de internacionalização, o estudo desenvolvido propiciou um intercâmbio entre instituições parceiras: UFF - UFOPA, tendo em vista os interesses comuns de pesquisa entre os envolvidos. Isso possibilitou o deslocamento de pesquisadores (supervisora de pós-doutorado) em diferentes regiões geográficas para participar de atividades de campo, em termos de pesquisa conjunta, além de atividades acadêmicas tais como a participação em bancas de defesa de mestrado e apresentações realizadas em eventos científicos.

Esta parceria resultou na possibilidade de fluxos de publicações associados aos Programas de vínculo dos integrantes das pesquisas. Com isso, a difusão de produção acadêmica entre instituições foi garantida, como, por exemplo, a publicação de artigos dos integrantes do GETUFF, em um dossiê sobre ensino de ciências e matemática, organizado pela Revista Êxitus, vinculado ao PPGE-UFOPA.

A experiência decorrente do pós-doutorado foi importante para a formação das pessoas envolvidas no processo, pois possibilitou várias aquisições em termos de conhecimento. Em primeiro lugar, a pesquisa em Etnomatemática tem cada vez mais utilizado referenciais pertencentes a outras áreas de conhecimento, o que tem contribuído para uma amplitude maior de discussões e reflexões sobre a natureza do conhecimento 
matemático e, mais particularmente, das ideias matemáticas ou práticas socioculturais presentes em diferentes contextos culturais.

Além disso, o acesso a referenciais alternativos permitiu conhecer pontos de vistas, perspectivas e experiências decorrentes da natureza do pensamento antropológico e das relações sociais envolvidas em processos informais de aprendizagem. Estas interfaces têm importância fundamental para compreender a natureza do pensamento e dos processos e técnicas envolvidas em práticas sociais. Estabelecem conexões de reflexões sobre como agentes de cognição estão efetivamente operando em um plano de formação de ideias matemáticas, baseada em técnicas e processos absorvidos e filtrados em um grupo sócio-cultural, localizado em uma região geográfica.

O contato com outros ambientes de ensino e de formação acadêmica, permitiu a troca de experiências e a discussão de especificidades existentes em diferentes locais de contexto regional, gerando a socialização de conhecimentos, experiências e referenciais utilizados, em estudos de pesquisa social. Isso correu, não apenas no nível nacional, mas internacional, e tais experiências foram refletidas em escritas e apresentações de trabalhos em diferentes eventos, em diferentes regiões do Brasil e no Exterior.

Pela primeira vez obteve-se uma experiência de internacionalização, o que mostra a importância desse tipo de estágio pós-doutoral, particularmente pelos contatos obtidos e experiências compartilhadas e socializadas.

Tal processo formativo, certamente tem um efeito de repercussão positivo para os que estão envolvidos neste processo. Particularmente a experiência adquirida no pós-doutorado possibilitou uma discussão mais aprofundada com os alunos matriculados nas disciplinas de graduação e pósgraduação, das Instituições envolvidas. Os debates desenvolvidos e troca de experiências entre pessoas de diferentes níveis e contextos educacionais forneceu subsídios para a reflexão envolvendo os estudos com base na 
Etnomatemática, contribuindo significativamente para os processos formativos dos participes do GETUFF.

As reuniões do grupo GETUFF bem como as ações envolvidas com a participação dos seus integrantes permitiu a expansão de possibilidades de investigações e pesquisas associadas. As discussões realizadas, os estudos e leituras desenvolvidas, possibilitaram uma compreensão maior do campo de expansão envolvendo as práticas socioculturais, revestidas de práticas envolvendo ideias matemáticas.

Além disso, os propósitos do GETUFF coincidem com os propósitos de um grupo voltado para os processos de formação de pesquisadores comprometidos com a Educação, o qual reflete a seriedade e a responsabilidade social do mesmo. Estes propósitos, certamente fornecem um acréscimo de incorporação de referenciais e estudos de efeito, voltados para as orientações em andamento, indicando assim um impacto positivo nos processos formativos dos envolvidos, em nível de graduação e pósgraduação. Consequentemente, estas ações contribuem, sem dúvida, para o aumento da qualidade da pesquisa em Educação e mais, especificamente, das pesquisas em Educação Matemática, especialmente as que estão relacionadas com a Etnomatemática.

\section{O papel formador dos grupos de pesquisa}

Olhar retrospectivamente para as experiências vivenciadas no contexto de um grupo de pesquisa, ao longo de muitos anos, certamente é um exercício que possibilita algumas reflexões sobre o papel desses grupos, assim como sobre as relações entre a Etnomatemática e os processos formativos dentro desta perspectiva.

Uma formação que parte de um enfoque etnomatemático é um processo contínuo de reflexão sobre a ação, devendo necessariamente considerar as experiências dos integrantes do grupo, seus saberes práticos e teóricos, seus valores (MONTEIRO, OREY \& DOMITE, 2004). Desenvolve na pessoa "a 
sensibilidade para o outro e suas formas de representar o mundo, exigindo pensar no conhecimento holisticamente e de maneira contextualizada, levando em conta as reações desiguais de poder na dinâmica cultural dos processos educativos" (FANTINATO, REZENDE \& DIAS, 2009, p. 56).

Os grupos de pesquisa em Etnomatemática também destacam-se como importante espaço de formação de novos pesquisadores. Que saberes fazem parte do repertório compartilhado de nossos grupos? Ao trabalharmos em Etnomatemática num constante processo de reconhecimento de saberes de outros, não estaríamos também reconhecendo nossos próprios saberes de pesquisadores dessa abordagem, e com isso, definindo nossa identidade? Como a formação na perspectiva etnomatemática consiste num processo contínuo e recíproco de legitimação de saberes (FANTINATO, REZENDE \& DIAS, 2009), parece que nossos grupos de estudos e pesquisa da área, e o GETUFF em particular, têm efetivamente desempenhado este papel formador.

\section{Referências}

ANDRE, M. Desafios da pós-graduação e da pesquisa sobre formação de professores. Educação \& Linguagem, n. 15, p. 43-59, jan./jul. 2007.

BOGDAN C.; BIKLEN S. K. Investigação qualitativa em educação. Portugal: Porto Editora, 1994.

CHAMOUX M. N. La transmission des savoir-faire : un objet pour l'ethnologie des techniques. Techniques et cultures. Bulletin de l'équipe de recherche 191, 3, p. 4683, 1978.

CRUZ M. C. Saberes do campo presentes em uma horta circular: uma pesquisa etnomatemática. 2016. 83 f. Qualificação de Mestrado (Mestrado em Educação). Faculdade de Educação. Universidade Federal Fluminense. Niterói/RJ.

D’AMBROSIO U. Etnomatemática: elo entre as tradiçôes e a modernidade. Belo Horizonte: Autêntica, 2001. (Coleção Tendências em Educação Matemática).

Etnomatemática e Educação. In: KNIJNIK, G.; WANDERER, F.; OLIVEIRA, C. J. (Orgs). Etnomatemática: currículo e formação de professores. Santa Cruz do Sul: EDUNISC, 2004. p. 39-52. 
Sociedade, cultura, matemática e seu ensino. Educação e Pesquisa, São Paulo, v.31, n.1, p. 99-120, jan/abr. 2005.

DASEN, P.R. Education informelle et processus d'apprentissage. In: P. R. DASEN \& A. AKKARI. Pédagogies et Pédagogues du Sud. Paris : L'Harmattan, 2004, p. 2352.

FANTINATO M. C. (Org.) Etnomatemática : novos desafios teóricos e pedagógicos. Niterói : Editora da UFF, 2009.

; REZENDE, W. M. \& DIAS, A. I. S. A Etnomatemática, a Matemática e a História na formação do professor de Matemática. Movimento n. 14. Niterói: EdUFF, 2009.

\& MAFRA, J. R. Techniques and learning process of craftswomen in Brazil. In: Ethnomathematics and its Diverse Approaches for Mathematics Education. ROSA M. Et al. (Eds.) Switzerland, Hamburg/Germany: Springer International Publishing. 2017. (ICME-13 Monographs).

; FREITAS A. Etnomatemática: concepções, dinâmicas e desafios. São Paulo: Paco Editorial, 2018.

FERREIRA, N. S. A. As pesquisas denominadas "estado da arte". Educação \& Sociedade, Campinas v. 23, n. 79, p. 257-272, ago. 2002.

FIORENTINI, D. Pesquisar práticas colaborativas ou pesquisar colaborativamente? In: BORBA, M. \& ARAÚJO, J. Pesquisa qualitativa em educação matemática. Belo Horizonte: Autêntica, 2004, p. 47-76.

FRANÇOIS, K.; MAFRA, J. R.; FANTINATO M. C; VANDENDRIESSCHE E. Local Mathematics Education: The Implementation of Local Mathematical Practices into the Mathematics Curriculum. In. Philosophy of Mathematics Education Journal ERNEST P. (Ed.) N. 33 January, 2018. On-line. Disponível em: http://socialsciences.exeter.ac.uk/education/research/centres/stem/publications/pme j/pome33/index.html. Acesso em: 22/01/2018.

GREENFIELD P. Cultural change and human development. New directions for child and adolescent development. n. 83, p. 37-59, Spring 1999.

LACERDA et al. A importância dos eventos científicos na formação acadêmica: estudantes de biblioteconomia. Revista $A C B$. Biblioteconomia em Santa Catarina, Florianópolis, v.13, n.1, p.130-144, jan./jun., 2008. Disponível em: <http://revista.acbsc.org.br/racb/article/viewFile/553/678>. Acesso em 16/01/2018.

LAVE J.; WENGER E. Situated learning: Legitimate peripheral participation. Cambridge: Cambridge University Press, 1993, cap.1 e 2, p. 27-58.

LÜDKE, M.; ANDRÉ, M. E. D. Afonso. Pesquisa em educação: abordagens qualitativas. 5. ed. São Paulo: EPU, 1986. 
MAFRA. J.R. Uma contribuição social e antropológica para a pesquisa em etnomatemática. 37a Reunião Nacional da ANPED. Florianópolis/SC. 2015.

MAFRA, J. R.; FANTINATO M. C. Artesãs de Aritapera/PA: técnicas e processos em uma perspetiva Etnomatemática. Revista Latinoamericana de Etnomatemática, 9(2), p. 180-201. 2016.

MEIRA, C. J. Os saberes das celas: um estudo etnomatemático com jovens e adultos em contexto de privação de liberdade. 2015. 119 f. Dissertação (Mestrado em Educação) - Faculdade de Educação. Universidade Federal Fluminense.

MONTEIRO A.; OREY, D.; DOMITE, M. C. Etnomatemática; papel, valor e significado. In: RIBEIRO, J.P. M.; DOMITE, M. C. S.; FERREIRA, R. (orgs). Etnomatemática: papel, valor e significado. Saõ Paulo: Zouk, 2004.

MOREIRA D. \& PIRES G. O processo educativo das crianças ciganas e aprendizagem da matemática. In: A. I. AFONSO (Org.) Etnografia com ciganos: diferenciação e resistência cultural. Lisboa: Edições Colibri, 2012.

PASSOS, C.. M. Condições de produção e legitimação da Etnomatemática. 2017. Tese de doutorado. (Programa de Pós-graduação em Educação). Universidade Federal de São Carlos, 2017.

THEES, A.; FANTINATO, M. C.; MEIRA, C.; ANDRADE, E. L. W.; SANTOS, F. L.M.; SOARES, G. A. Retrospectiva e perspectivas do Grupo de Etnomatemática da UFF - GETUFF. Montivideu. Actas... del VII CIBEM, 2013, p.7926-7933.

VANDENDRIESSCHE E.; MAFRA J.R.; FANTINATO M.C.; FRANÇOIS K. How Local are Local People? Beyond Exoticism. Proceedings of the Ninth International Mathematics Education and Society Conference. CHRONAKI A. (Ed.) Anais... Vol. 2. Volos, Greece: University of Thessaly Pess, 2017. p. 956-967. 